TAPROBANICA, ISSN 1800-427X. November, 2021. Vol. 10, No. 02: pp. 140-145.

(C) Research Center for Climate Change and Department of Biology, Faculty of Mathematics \& Natural Sciences, University of Indonesia, Depok 16424, INDONESIA.

http://www.taprobanica.org

https://doi.org/10.47605/tapro.v10i2.269

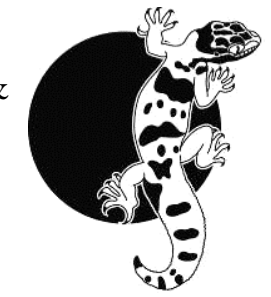

\title{
A response to Narayanan et al. (2021) "On the validity of the recently described bush frog Raorchestes kollimalai Gowande, Ganesh \& Mirza, 2020" published in Zootaxa
}

Submitted: 26 August 2021

\author{
Zeeshan A. Mirza ${ }^{1, *} \&$ Gaurang Gowande ${ }^{2,3}$ \\ ${ }^{1}$ National Centre for Biological Sciences, TIFR, GKVK Campus, Bellary Rd., Bangalore 560065, India \\ ${ }^{2}$ Department of Biodiversity, Abasaheb Garware College, Pune, Maharashtra 411004, India \\ ${ }^{3}$ Department of Biotechnology, Fergusson College, Pune, Maharashtra 411004, India \\ *Corresponding author. E-mail: snakeszeeshan@gmail.com
}

Narayanan et al. (2021) make extremely grave allegations of 'misrepresentation of data' by Gowande et al. (2020). Narayanan et al. (2021) found serious flaws in the publication dealing with the description of Raorchestes kollimalai by Gowande, Ganesh \& Mirza (2020). The authors state that Gowande et al. (2020) overlooked many errors, which consequently raises questions on the validity of $R$. kollimalai. We here respond to their comments, dispel their false allegations of misrepresentation of data and provide evidence for the validity of Raorchestes kollimalai.

A common practice while writing a response/critique of a published paper is to contact the editor of the journal in which the paper was published, which, in this case, is TAPROBANICA. However, Narayanan et al. (2021) chose to publish their critique in a different journal, ZOOTAXA and thus, were unaware of the erratum to the paper on the new species. The erratum that was published in a recent issue of Taprobanica (Gowande et al. 2021) resolves some of the concerns raised by Narayanan et al. (2021). These concerns were a mistake on the part of Gowande et al. (2020) but by no means did the authors intend to hide or misrepresent data as accused by Narayanan et al. (2021).

"Issues in molecular analysis": Phylogenetic relationships within the genus Raorchestes Biju, Shouche, Dubois et al., 2010 have been well studied (Biju et al. 2010, Garg et al. 2021, Vijayakumar et al. 2014, 2016). These studies have not only provided a sound understanding of the phylogenetic relationships among these rhacophorid anurans, but also the underlying mechanisms of diversification. Extensive sampling of bush frogs by Vijayakumar et al. $(2014,2016)$ has led to the discovery of several new species and the data further hinted at the presence of cryptic undescribed lineages. Gowande et al. (2020) recently described one such lineage as Raorchestes kollimalai from the southern Eastern Ghats. The description of the new species was based on morphology and molecular data of three specimens. Gowande et al. (2020) generated 16S rRNA sequences for the three specimens and showed that the species was $1.2-1.9 \%$ divergent from its sister taxon, $R$. charius (Rao, 1937). The 16S dataset was subjected to phylogenetic analysis through the Maximum Likelihood (ML) and Bayesian Inference (BI) approach. Based on 16S sequence identity, Gowande et al. (2020) considered the specimen CESF069 (housed in the museum of the Centre for Ecological Sciences, Indian Institute of Sciences, Bangalore (CES)) conspecific with the new species and 
included the sequence generated by Vijayakumar et al. (2014) from an unknown locality in the analysis. Vijayakumar et al. $(2014,2016)$ cumulatively generated sequences of five genes (four mitochondrial and one nuclear) from the specimen CESF069. Molecular phylogenetic reconstructions by Vijayakumar et al. $(2014,2016)$ recovered this species as a member of the Charius clade and was referred to as an unidentified cryptic species [R. UI CESF069 in Figure 3 page 459 of Vijayakumar et al. (2014) and Raorchestes UI CESF069 in Figure 2 page 4 of Vijayakumar et al. (2016)]. Gowande et al. (2020), at the time of their publication, were not aware of the precise collection locality of the Raorchestes specimen CESF069, although Gowande et al. (2020) used the sequences derived from this specimen. A recent inquiry with the CES museum about the locality of the specimen CESF069 not only confirmed the speculation of the Eastern Ghats origin of the specimen by Gowande et al. (2020) but further works in favour of them in being conspecific to R. kollimalai as the specimen was collected from Kolli Hills, the type locality of $R$. kollimalai. The revelation of the collection locality of the specimen CESF069 further supports that the species is genetically divergent and is distinct from $R$. charius in exhibiting an uncorrected $p$-distance of $5 \%$ for the mitochondrial NADH dehydrogenase subunit 1 gene (Table 1 on page 166 of Gowande et al. 2020). As stated in the 'Molecular analysis' of Material and Methods section on page 165 and Results section page of Gowande et al. (2020) and further, reiterated above, the dataset subjected to phylogenetics was for the gene 16S rRNA and there is no mention of the concatenation of $16 \mathrm{~S}$ and ND1. The question of whether the two genetic regions were concatenated does not arise at all. The sequences of ND1 region that were generated by Vijayakumar et al. (2014) were only used to assess pairwise sequence divergence (in the 'Molecular analysis' of Material and Methods section and Table 1 on page 166) and were not subjected to phylogenetic analysis.

Furthermore, Gowande et al. (2020) deal with ND1 in the 'Molecular analysis' of Material and Methods section on page 166 only while specifying the methodology for the calculation of uncorrected p-distance (divergence). Likely Narayanan et al. (2021) did not read the paper thoroughly and assumed that the two genes were concatenated. Based on their assumptions, they blatantly accused Gowande et al. (2020) of assigning a single sequence evolution model to the assumed concatenated phylogenetic analysis. To those who are versed in molecular phylogenetics, clades with bootstrap support $>70$ are considered well supported (Hillis \& Bull 1993) and we consider branch bootstrap support of 91 sufficient to infer phylogenetic relationships. Gowande et al. (2020) reported that the support for the clade is 100 whereas it is 91 , which is not considered low. However, Narayanan et al. (2021) used the word "only" to describe the high bootstrap support of 91 recovered in the ML phylogeny (Fig. 1 of Gowande et al. 2020) raising doubts about their understanding of molecular phylogenetics. Naranayan et al. (2021) bring to our notice the polytomy inferred $[R$. kollimalai $+R$. charius] as the sister clade of $R$. honnametti Gururaja, Priti, Roshmi \& Aravind, 2016 by Gowande et al. (2020). Indeed there is a polytomy, which is likely an artifact of lack of molecular markers as the two species are recovered sisters in Vijayakumar et al. $(2014,2016)$.

"Issues in morphometric comparisons": Narayanan et al. (2021) state that Gowande et al. (2020) used a limited set of morphological characters to delimit $R$. kollimalai from members of the Charius clade. It appears that the authors failed to notice that the described species belongs to a cryptic species complex, which is stated clearly in the title of the paper. Furthermore, the term 'cryptic' appears at least eight times in Gowande et al. (2020), including the title. This further goes to show that Narayanan et al. (2021) did not read Gowande et al. (2020) thoroughly; otherwise, the word cryptic could hardly be missed. Cryptic species in most cases are indistinguishable from their related species based on a single line of evidence, like morphology (Delić et al. 2017). Multiple lines of evidence in combination must be employed to disentangle such species (Ambekar et al. 2020, Mirza et al. 2018), which comprises a substantial proportion of biodiversity (Pfenninger \& Schwenk 2007). Gowande et al. (2020) used characters that were differing or overlapping in combination with other lines of evidence and did not just base their description of the new species on the morphology of three specimens. The authors further conveniently state that the other two characters used to distinguish $R$. kollimalai from $R$. charius, IUE/SVL $0.14-0.16$ vs. $0.11-0.13$; TL/SVL $0.32-0.43$ vs. $0.44-0.51$ is a continuous range without presenting any new data.

Principal Component Analysis (PCA) and Discriminant Function Analysis (DFA) are analyses that are employed to reduce dimensionality in large datasets. A ratio of 10 data points per variable is recommended (Pallant 2001) and such a large sample size is generally discouraged in a 
threatened landscape such as the Eastern Ghats. Despite this, on the recommendations of Narayanan et al. (2021), we performed the multivariate PCA based on existing data of the type specimens of $R$. kollimalai and data for other members of the Charius clade taken from Priti et al. (2016) and Biju \& Bossuyt (2009). The exploratory analysis did not recover distinct clusters corresponding to the currently recognized members of the Charius clade (PC1 \& PC2 explain 57.67+14.72\% of the variance observed Fig. 1, Appendix I). Gowande et al. (2020) did not perform PCA due to the low sample size. Narayanan et al. (2021) appear to be unfamiliar with the theoretical assumptions of these statistical analyses and given their way, they would likely merge all members of the Charius clade into a single species based on the results of PCA (Fig. 1). As rightly stated by Narayanan et al. (2021), Gowande et al. (2021) did make an error while reporting the range for the character HW/SVL for the species $R$. kollimalai, which was used as a diagnostic character in the description of the species. Nonetheless, this error was corrected in the published erratum.

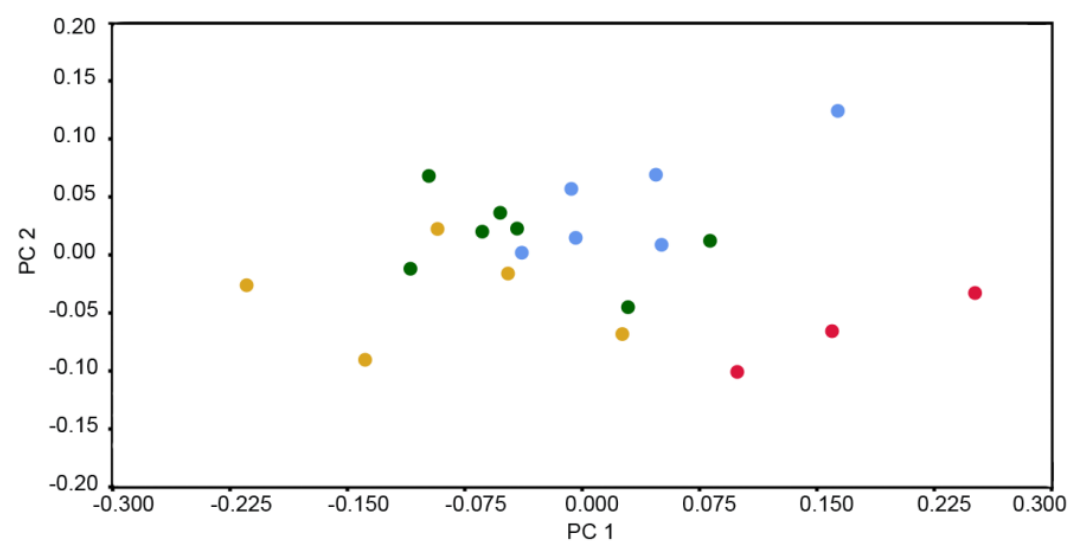

Figure 1. PCA plot of members of the Charius clade, PC1 and PC2 explain $57.67+14.72 \%$ of the variance; each solid circle corresponds to Raorchestes species: R. kollimalai (red), R. charius (green), R. griet (Bossuyt, 2002) (gold), and $R$. honnametti (blue). Note: only adult male specimens were used for the analysis.

"Issues in acoustic analysis" Frog calls can vary greatly and several studies show that the same species may show variation in the call with respect to changes in temperature, altitude and even other ambient sounds. Thus, we agree with Narayanan et al. (2021) and refrain from arguing on the acoustic analysis. The call may not be used to distinguish $R$. kollimalai from other members of the Charius clade, but can be used to distinguish it from congeners (Fig. 2):

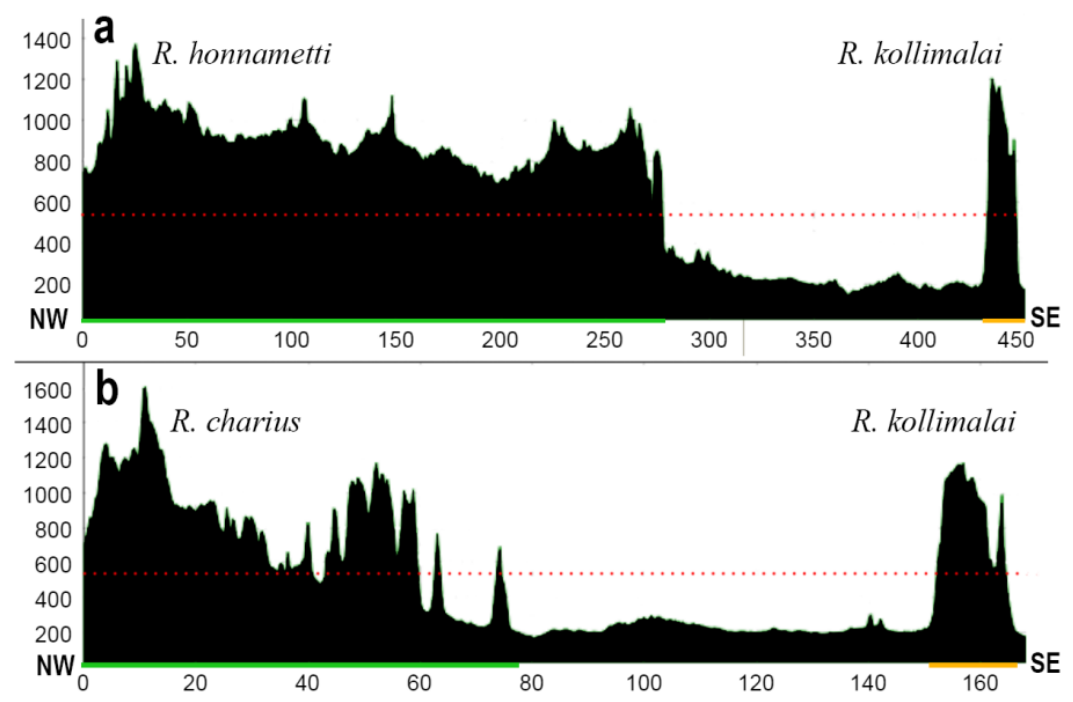

Figure 2. Schematic representation of elevational profile of the Western Ghats (green bar) and the isolated Kolli Hills of the Eastern Ghats (orange bar) showing the distribution of $R$. kollimalai. (a) Biligiri Rangaswamy Tiger Reserve to Kolli Hills, and (b) Bhadra Tiger Reserve to Kolli Hills. The red dotted line shows distribution limit of $R$. kollimalai; $\mathrm{NW}=$ northwest, $\mathrm{SW}=$ southwest. 
"Biogeographic analysis": The Western Ghats is a 1600km long hill range with two distinct gaps, the Palghat gap and the Shencottah gap. These gaps are dry zones which play vital roles in acting as barriers for gene flow across these gaps for wet zone species (Abraham et al. 2013, Joshi \& Karanth 2013, Robin et al. 2017, Vijayakumar et al. 2016). Similarly, the Western and Eastern Ghats are separated by intervening arid plains that are inhospitable to wet adapted organisms such as Raorchestes. Some of the low genetic divergence within such organismal groups that inhabit these two mountain complexes can be explained by the presence of conducive vegetational causeways that existed until quite recently. The wet evergreen vegetation in the Eastern Ghats is also quite similar in composition to the Western Ghats and is likely to be a Pleistocene relict (Mani 1974; Vijayakumar et al. 2016). Several Raorchestes species may have a broad distribution range within the wet zones of the Western Ghats as much as $\sim 400 \mathrm{~km}$ for $R$. charius (Narayanan et al. 2021). However, R. kollimalai appears to be restricted to wet zone forests of elevation above $500 \mathrm{~m}$ and may be found in adjacent hill ranges of the Eastern Ghats. Data for ND1 gene further confirms that $R$. kollimalai is divergent from the Western Ghats population and likely a result of a lack of gene flow due to the low elevation dry zone. Biligiri Rangaswamy Tiger Reserve is the type locality of $R$. honnametti that is closest to the type locality of $R$. kollimalai. A schematic representation of the elevational profile of a straight line from Biligiri Rangaswamy Tiger Reserve to Kolli Hills shows that the latter is separated by a wide, dry lowland zone that is uninhabitable for Roarchestes (Fig. 2). Surveys conducted in the southern Eastern Ghats confirm that Roarchestes is absent from areas lower than $500 \mathrm{~m}$, which are too dry and hot for these rhacophorid frogs (Gowande et al. 2020, Akshay Khandekar personal observation). The dry zone separates the populations from the Western Ghats and the Eastern Ghats and rejecting this biogeographic scenario hints at a poor understanding of biogeography by Narayanan et al. (2021).

Shallow divergence: Narayanan et al. (2021) draw attention to the case of shallow genetic divergence between R. bombayensis (Annandale, 1919) and R. sanctisilvaticus (Das \& Chanda, 1997), which are broadly distributed across the Western Ghats and the Eastern Ghats respectively (Biju \& Bossuyt 2009; Garg et al. 2021; Mirza et al. 2019). They credit Garg et al. (2021) for highlighting the shallow divergence between the species. However, the findings were first presented by Mirza et al. (2019) and confirmed by Garg et al. (2021). This suggests a poor literature review by Narayanan et al. (2021). Garg et al. (2021) and Mirza et al. (2019) retain the two species despite low divergence, as data from throughout the range would be required to consider them synonymous. Narayanan et al. (2021) have cited Garg et al. (2021) and yet selectively ignore this cautious approach. A review of pdistance for 16S rRNA gene across Roarchestes shows that sister species show a divergence as low as $1 \%$ in the Tinniens clade (Vijayakumar et al. 2014) thereby suggesting that species cannot be synonymized just based on low genetic divergence. Evolutionary rates vary across lineages within a gene tree (Britten 1986) and hence one cannot draw p-distance based cut-offs to delineate species without understanding the structure of the entire population.

Discussion and Conclusion: Synonymization of a species should be done with the utmost caution and with substantial data to support the action. Narayanan et al. (2021) could have examined the type specimens of $R$. kollimalai to ascertain its validity, but clearly, their interest was not in the species or taxonomic stability. In many cases, valid taxa are considered synonymous just as the new species description uses overlapping characters or is based on low sample size or low genetic divergence. The synonymization of $R$. kollimalai by Narayanan et al. (2021) appears to be the inappropriate, as the authors have not read the original description in detail, have conducted a poor literature review regarding the genus Raorchestes, and have a poor understanding of the concepts of cryptic species, biogeography and phylogenetics. The correspondence by Narayanan et al. (2021) further lacks species authority for 11 species out of the 12 listed therein reflecting a complete disregard to the International Code of Zoological Nomenclature (ICZN 1999) and general norms in classical taxonomy. The false allegation of misrepresentation of data without any valid proof negatively affects the credibility of the authors, and synonymy of the species, which is valid, would deprive the species of conservation management attention. If the authors' intent was academic, they would have examined the type specimens, visited the type locality to gather more specimens, and call data to assess the validity of the species like Mirza et al. (2019) and Kalki et al. (2020) did, but they choose instead to criticize Gowande et al. (2020), which suggests a personal attack. As demonstrated above, Narayanan et al. (2021) do not provide any evidence that the data presented by Gowande et al. (2020) was "misrepresentation" in any way and their synonymization of the species is baseless. We 
propose with conviction to retain $R$. kollimalai as a valid species. To summarize, no phrase is more approriate than "Those who live in glass houses should not throw stones".

The editors of Zootaxa who published the correspondence by Narayanan et al. (2021) should have asked the authors to submit their critique to Taprobanica, in which the original description of $R$. kollimalai was published. Furthermore, Zootaxa refused to accept a response from us and demanded new data to support our claims. Authors have the right to respond to criticism and false allegations but by denying acceptance of our response, the journal Zootaxa violated ethical practices. On the webpage of Zootaxa, the instructions to submission states "Commentaries on published papers are intended for scholarly exchange of different views or interpretations of published data and should not contain personal attack; authors of concerned papers may be invited to reply to comments on their papers", but the handling editor refused to accept our response, demanding additional data and reanalysis. It is quite surprising that the handling editor accepted the correspondence by Narayanan $e t$ al. (2021) without any new data and more so with grave false allegations without any justification. It is a pity that it even passed peer review, merely reflecting negligence on the part of the editor and reviewers. It is hoped that journals that publish critiques and or comments, ensure due diligence on their part as to not encourage violation of publication ethics that would severely affect the scientists involved and eventually the science.

We would like to acknowledge the national and international herpetological community for their support and suggestions on these matters. Special thanks to Varad Giri, R. Chaitanya, A.A. Thasun Amarasinghe, Aamod Zambre, Harshal Bhosale, Saunak Pal, Yatin Kalki and Akshay Khandekar for valuable comments.

\section{Literature cited}

Abraham, R.K., R.A. Pyron, B.R. Ansil et al. (2013). Two novel genera and one new species of treefrog (Anura: Rhacophoridae) highlight cryptic diversity in the Western Ghats of India. Zootaxa, 3640(2): 177-199.

Ambekar, M., A. Murthy, and Z.A. Mirza (2020). A new species of fan-throated lizard of the genus Sitana Cuvier, 1829 (Squamata: Agamidae) from northern Karnataka, India. Bonn Zoological Bulletin, 69(2): 157-164.

Biju, S.D. and F. Bossuyt (2009). Systematics and phylogeny of Philautus Gistel, 1848 (Anura, Rhacophoridae) in the Western Ghats of India, with descriptions of 12 new species. Zoological Journal of the Linnean Society, 155(2): 374-444.

Biju, S.D., Y. Shouche, A. Dubois et al. (2010). A ground-dwelling rhacophorid frog from the highest mountain peak of the Western Ghats of India. Current Science, 98(8): 1119-1125.

Britten, R.J. (1986). Rates of DNA sequence evolution differ between taxonomic groups. Science, 231: 1393-98.

Delić, T., P. Trontelj, M. Rendoš, and C. Fišer (2017). The importance of naming cryptic species and the conservation of endemic subterranean amphipods. Scientific Reports, 7(1): 1-12.

Garg, S., R. Suyesh, S. Das et al. (2021). An integrative approach to infer systematic relationships and define species groups in the shrub frog genus Raorchestes, with description of five new species from the Western Ghats, India. PeerJ, 9: e10791.

Gowande, G., S.R. Ganesh, and Z.A. Mirza (2021). Erratum for "A new cryptic species of bush frog (Amphibia: Anura: Raorchestes) from the Southern Eastern Ghats, India." Taprobanica, 10(1): 79.

Gowande, G.G., S.R. Ganesh, and Z.A. Mirza (2020). A new cryptic species of bush frog (Amphibia: Anura: Raorchestes) from the Southern Eastern Ghats, India. Taprobanica, 9(2): 164-173.

Hillis, D.M. and J.J. Bull (1993). An empirical test of bootstrapping as a method for assessing confidence in phylogenetic analysis. Systematic Biology, 42(2): 182-192.

ICZN (1999). International Code of Zoological Nomenclature, $4^{\text {th }}$ edition. International Trust for Zoological Nomenclature, London.

Joshi, J. and P. Karanth (2013). Did southern Western Ghats of peninsular India serve as refugia for its endemic biota during the Cretaceous volcanism? Ecology and evolution, 3(10): 3275-82.

Kalki, Y., S. Gowda, M. Agnivamshi et al. (2020) On the taxonomy and systematics of the recently described Lycodon deccanensis Ganesh, Deuti, Punith, Achyuthan, Mallik, Adhikari, Vogel, 2020 (Serpentes, Colubridae) from India. Evolutionary Systematics, 4(2): 109-118.

Mani, M. (1974). Ecology and biogeography in India. Dr. W. Junk B.V. Publishers, The Hague, The Netherlands. 
Mirza, Z.A., G. Gowande, R. Patil et al. (2018). First appearance deceives many: disentangling the Hemidactylus triedrus species complex using an integrated approach. PeerJ, 6: e5341.

Mirza, Z.A., P. Warekar, P.P. Mohapatra et al. (2019). Endangered or an artifact of unsound taxonomy? Case of the critically endangered bush frog Philautus sanctisilvaticus Das and Chanda, 1997. Zootaxa, 4683(4): 563-576.

Narayanan, S., R. Suyesh, and S. Das (2021). On the status and validity of the recently described Raorchestes kollimalai Gowande, Ganesh \& Mirza, 2020. Zootaxa, 4970(3): 597-600.

Pallant, J. (2001). SPSS survival manual: A step by step guide to data analysis using SPSS. Allen \& Unwin, Crows Nest.

Pfenninger, M. and K. Schwenk (2007). Cryptic animal species are homogeneously distributed among taxa and biogeographical regions. BMC Evolutionary Biology, 7(1): 121.

Priti, H., R. Roshmi, B. Ramya et al. (2016). Integrative Taxonomic Approach for Describing a New Cryptic Species of Bush Frog (Raorchestes: Anura: Rhacophoridae) from the Western Ghats, India. PLoS One, 11: $\mathrm{e} 0149382$.

Robin, V.V., C.K. Vishnudas, P. Gupta et al. (2017). Two new genera of songbirds represent endemic radiations from the Shola Sky Islands of the Western Ghats, India. BMC Evolutionary Biology, 17: 31.

Vijayakumar, S.P., K.P. Dinesh, M.V. Prabhu, and K. Shanker (2014). Lineage delimitation and description of nine new species of bush frogs (Anura: Raorchestes, Rhacophoridae) from the Western Ghats Escarpment. Zootaxa, 3893(4): 451-488.

Vijayakumar, S.P., R.C. Menezes, A. Jayarajan, and K. Shanker (2016). Glaciations, gradients, and geography: Multiple drivers of diversification of bush frogs in the western ghats escarpment. Proceedings of the Royal Society (Biological Sciences), 283(1836): 20161011.

Appendix I. Factor loadings on the principal component (PC) for each morphometric variable. For the abbreviations used, refer to Gowande et al. (2020)

\begin{tabular}{lrr}
\hline Character & PC 1 & PC 2 \\
\hline head width (HW) & 0.113200 & 0.270260 \\
head length (HL) & -0.034380 & 0.013691 \\
inner upper eyelid width (IUE) & 0.492430 & 0.194650 \\
maximum upper eyelid width (UEW) & 0.776470 & -0.434170 \\
snout length (SL) & 0.283750 & 0.624180 \\
eye length (EL) & 0.202620 & 0.110530 \\
forelimb length (FLL) & 0.084967 & -0.366620 \\
hand length (HAL) & 0.076693 & 0.259150 \\
shank length (ShL) & 0.065681 & 0.204910 \\
foot length (FOL) & 0.040295 & 0.234550 \\
\hline
\end{tabular}

